\title{
SwissDRG: ein Zwischenfazit
}

\section{Beatrix Meyer}

Leiterin Tarifdienst FMH und Bereich SwissDRG
In eineinhalb Jahren ist es bereits so weit: Dann werden die Spitäler ihre akutstationären Leistungen mit dem Fallpauschalensystem SwissDRG abrechnen. Wie ist der Stand der vorbereitenden Arbeiten, und welche Problempunkte gilt es noch zu lösen? Welche begleitenden sichernden Massnahmen braucht es für die Einführung? Nachdem kürzlich die SwissDRG-Version 0.3 veröffentlicht wurde, ist es ein guter Zeitpunkt, diesen Fragen auf den Grund zu gehen.

\section{Die neue SwissDRG-Version 0.3}

Die Bereitstellung der SwissDRG-Tarifstruktur Version 0.3 ist zweifelsfrei ein weiterer wichtiger Meilenstein auf dem Weg bis zur SwissDRG-Einführung im Jahr 2012. Durch zahlreiche Simulationen und Analysen konnte die SwissDRG AG die Güte des Systems verbessern. Allerdings sind im Hinblick auf die SwissDRG-Einführung und die zu erarbeitende definitive Einführungsversion 1.0 noch mehrere Problempunkte zu lösen.

Nachfolgend sind die aus Sicht der Ärzteschaft wichtigsten Punkte zusammengefasst, welche die FMH im Rahmen ihrer Stellungnahme zur SwissDRG-Version 0.3 an die SwissDRG AG eingereicht hat: die SwissDRG AG aber noch nicht umsetzen, weil der Grundsatzentscheid der Partner ausstehend und die Datenlage ungenügend ist. Zur Verbesserung der Datenlage hat die FMH einen Antrag an die SwissDRG AG eingereicht, damit die Spitäler wie in Deutschland die Möglichkeit von «ergänzenden Datenlieferungen» erhalten. Die Universitätsspitäler haben der FMH bereits die Bereitschaft signalisiert, diesen zusätzlichen Aufwand für ergänzende Datenerfassungen zu leisten. Anhand dieser Daten könnte die SwissDRG AG die Zusatzentgelte dann kalkulieren.

\section{Qualität der Kosten- und Leistungsdaten noch ungenügend}

Die Güte des SwissDRG-Systems hängt entscheidend von der Qualität der Kosten- und Leistungsdaten ab. Diese ist allerdings nach wie vor ungenügend. So mussten im Rahmen der Plausibilisierungen 23\% der von den Netzwerkspitälern gelieferten Datensätze aus der Stichprobe für die Kalkulation der SwissDRG-Version 0.3 ausgeschlossen werden. Zudem konnten für die SwissDRG-Version 0.3 nur $53 \%$ der DRGs anhand der Schweizer Daten kalkuliert werden. Für die restlichen DRGs mussten helvetisierte deutsche Kostengewichte

\section{Zusatzentgelte gewährleisten eine leistungsgerechte Vergütung für teure Produkte und Verfahren}

\section{Schaffung von Zusatzentgelten}

Das SwissDRG-System baut auf der Grundlage des deutschen DRG-Systems auf. Sehr gute Erfahrungen hat man in Deutschland mit dem Prinzip der sogenannten Zusatzentgelte gesammelt. Bei den Zusatzentgelten handelt es sich nicht um ein «zusätzliches» Entgelt, sondern um eine separate Vergütung ausserhalb der Fallpauschalen. Zusatzentgelte sind für die leistungsgerechte Vergütung von teuren Medikamenten, Blutprodukten, Implantaten und kostenintensiven Behandlungsmethoden unerlässlich. Sie tragen dazu bei, die Übersichtlichkeit des DRG-Systems zu bewahren und die Schaffung von unzähligen neuen DRG-Fallgruppen $\mathrm{zu}$ vermeiden. In der neuen SwissDRG-Version 0.3 wurden nur bei einem einzigen Verfahren, nämlich der Nierenersatztherapie, drei Zusatzentgelte geschaffen. In den Antragsverfahren 2008 und 2009 hatten die Fachgesellschaften jedoch bereits für zahlreiche weitere Anwendungsbereiche und Produkte die Schaffung von Zusatzentgelten beantragt. Diese Anträge konnte herangezogen werden. Die Qualität der Kostendaten ist u. a. deshalb ungenügend, weil die Spitäler die Kosten bisher nicht nach einer einheitlichen Methode erfassen. Das Qualitätsproblem der Leistungsdaten ist vor allem darauf zurückzuführen, dass die Verfahren in der aktuellen Version der Schweizerischen Prozedurenklassifikation $\mathrm{CHOP}$ in vielen Bereichen zu wenig differenziert abgebildet sind. Diese Problematik ist erst mit der nächstes Jahr gültigen Version CHOP 2011 gelöst, mit welcher die zahlreichen CHOP-Anträge der Fachgesellschaften umgesetzt sein werden. Die mit der CHOP 2011 erfassten Daten kann die SwissDRG AG dann im Jahr 2012/2013 kalkulieren und eine Vergütung der entsprechenden Leistungen erfolgt erstmals im Jahr 2014.

\section{Resultat des Antragsverfahrens}

Zur Verbesserung des Systems haben die medizinischen Fachgesellschaften und ärztlichen Dachverbände in den Jahren 2008 und 2009 über 1800 An- 
träge zum DRG-System und zu den Klassifikationen eingereicht. Davon waren 29 Anträge explizite DRGAnträge. Gemäss der SwissDRG AG waren 11 dieser 29 DRG-Anträge rechenbar. 5 DRG-Anträge wurden in der SwissDRG-Version 0.3 umgesetzt. Noch nicht in der SwissDRG-Version 0.3 enthalten sind die knapp 1800 Anträge der Fachgesellschaften und Dachverbände zur Prozedurenklassifikation CHOP aus den Antragsjahren 2008 und 2009. Diese werden zurzeit durch das Bundesamt für Statistik (BFS) für die neue, nächstes Jahr gültige CHOP-Version 2011 umgesetzt. Zur Begleitung der Antragsumsetzung hat die FMH 36 Gespräche mit dem BFS, den Fachgesellschaften, Dachverbänden und den FMH-Experten organisiert und inhaltlich unterstützt. Diese Gespräche waren sehr konstruktiv und die Mehrheit der Anträge konnten schliesslich umgesetzt werden. Auch dieses Jahr unterstützt die FMH die Fachgesellschaften wieder nach Kräften beim Antragsverfahren. Einsendeschluss für DRG-Anträge ist der 4. August 2010 und für Anträge zu den Klassifikationen der 20. September 2010.
Der Bundesrat hat den Tarifpartnern im Rahmen seiner Genehmigung der SwissDRG-Version 0.2 eine entsprechende Nachfrist gewährt. Bis Ende 2010 müssen die Tarifpartner Unterlagen betreffend Qualität, Monitoring und Schätzungen über die Auswirkungen des Tarifs nachreichen [3].

\section{Fazit: national einheitliche Übergangsregelung während der Einführungsphase erforderlich}

Die Analyse der neuen SwissDRG-Version 0.3 und der noch nicht gelösten Problempunkte zeigt vor allem eines deutlich: Es braucht zwingend eine Übergangsregelung zur Abfederung von Systemverzerrungen. Auf jeden Fall muss verhindert werden, dass gut arbeitende Spitäler finanzielle Verluste erleiden, nur weil ihr Behandlungsspektrum im SwissDRG-System ungenügend abgebildet ist. Entsprechende finanzielle Garantien sind deshalb notwendig. Eine leistungsgerechte Vergütung und eine gewisse Planungssicherheit sind für die Spitäler und ihre Fachabteilungen gerade in der Einführungsphase zentral.

\section{Eine national einheitliche Übergangsregelung ist notwendig, damit gut arbeitende Spitäler nicht systembedingt Verluste schreiben}

Keine separate Vergütung von Frührehabilitation Aus Sicht der FMH ist der Vorschlag, die Frührehabilitation einschliesslich der geriatrischen frührehabilitativen Komplexbehandlung aus dem DRG-System auszulagern und über einen separaten Rehatarif zu vergüten, höchst problematisch. Rechtzeitige, eng mit akutmedizinischen und chirurgischen Massnahmen integrierte frührehabilitative Massnahmen im Akutspital sind im Sinne der Patienten und integraler Bestandteil der akutsomatischen Versorgung. Darüber hinaus verkürzen sie die anschliessenden Aufenthalte in der Rehabilitation. Deshalb setzt sich die FMH dafür ein, dass die Frührehabilitation nicht aus dem SwissDRG-System ausgegliedert wird.

\section{Weitere zu klärende Fragen}

Verschiedene weitere in der Schweizerischen Ärztezeitung bereits beschriebene Fragen sind noch ungeklärt, wie z. B. die rasche Vergütung von Innovationen [1] oder die Finanzierung der ärztlichen Weiterbildung [2]. Aber auch die Umsetzung der gesetzlichen Vorgabe, dass die Investitionen und Anlagenutzungskosten über SwissDRG vergütet werden müssen, bereitet angesichts der uneinheitlichen Anlagebuchhaltungen der Spitäler Sorge [1]. Nicht geklärt sind insbesondere Fragen des Qualitätscontrollings und des Monitorings.
Wichtig ist, dass diese Übergangsregelung zur finanziellen Abfederung von Systemverzerrungen während der Einführungsphase national einheitlich definiert wird. Individuell vereinbarte Massnahmen auf Ebene der Tarifverträge - wie dies verschiedene Partner vorschlagen - würden ungleiche Spiesse schaffen. Wettbewerbsverzerrungen wären die Folge. Im Hinblick auf die oben genannten ungelösten Fragen sollte diese national einheitliche Übergangsregelung mindestens für die Einführungsphase 2012/2013 veranlagt werden. Eine rasche Einigung auf eine national einheitliche Übergangsregelung ist wichtig, genauso wie die stetige Weiterarbeit und Lösungsfindung zu den oben genannten Problempunkten. Die FMH jedenfalls bietet wie in der Vergangenheit ihre konstruktive Mitarbeit und Unterstützung an.

\section{Literatur}

1 Vgl. Cuénoud P.-F. SwissDRG - ist die Einführung am 1. Januar 2010 realistisch? Schweiz Ärztezeitung. 2010;91(17):657.

2 Vgl. Giger M. Bundesrätliche Verordnung gefährdet ärztlichen Nachwuchs. Schweiz Ärztezeitung. 2008;89(46):1981.

3 Vgl. www.news.admin.ch/message/ ?lang=de\&msg-id=33769 